\title{
INTRODUCTION TO THE SPECIAL ISSUE ON LEARNING, OPTIMIZATION, AND THEORY OF G-NETWORKS
}

\author{
Nihal Pekergin \\ LACL, Faculté des Sciences et Technologie, Université de Paris-Est Créteil, 61 avenue du Général de \\ Gaulle 94010 Créteil, France \\ E-mail: nihal.pekergin@u-pec.fr
}

\begin{abstract}
We introduce the special issue on "Learning, Optimization, and the Theory of G-Networks" of the journal Probability in the Engineering and Informational Sciences that appears in 2019. We first outline some of the applications and developments of G-Networks which motivate the ongoing interest for this area, including some areas which could not be covered in this special issue. We then briefly discuss the contributions presented in the ten papers that are published in this special issue in the context of related work.
\end{abstract}

Keyword: probabilistic networks

\section{INTRODUCTION TO THE SPECIAL ISSUE}

Within the field of probabilistic networks and queueing theory, the area of G-Networks began with the papers $[8-10,34]$ which introduced the "primitive" model of queueing networks with negative and positive customers and their product form steady-state solution. Starting from the theory of Queueing Networks with Product Form Solutions [31,32,53], G-Networks developed rapidly with the help of several generalizations $[11,38]$.

Simultaneously with the development of the initial theoretical results, diverse applications emerged in distinct areas such as:

- Self-aware and autonomic computer networks [3,5,7,20,40], including the concept of "energy aware routing" [29] and the performance of networks where packets are opportunistically [42] or autonomously routed [16,39].

- Adaptive management of Cloud systems [54,55],

- Machine learning (ML) $[12,27]$ and Deep Learning with Random Neural Networks [36], and their applications to image and object recognition,

- The modeling of Gene Regular Networks [18,45,52] and their exploitation for the detection of anomalies that can lead to disease [46,46-49],

- The link between G-Networks and Genetic Algorithms [14,15] and its application to network routing [41]. 
- The new concept of Energy Packet Networks for the optimization of energy consumption in ICT and systems where the inflow of energy, and the consumption of energy, are both intermittent and can be represented by random processes $[1,21-26]$. These also gave rise to new product form solutions [44] and applications [51].

- Cybersecurity $[4,17,28,35,50]$ with its implications in the security of Cyber-Physical Systems [35].

- Queueing networks with tightly coupled behaviors [2] that arise in the study of distributed systems [33], and the ability of G-Networks to compute the steady-state behavior of distributed systems that operate under the effect of dynamic control algorithms [30].

Let us also mention other work which develops a product form solution for an (economic) market composed of $N$ English auctions [19] where customers are buyers that arrive according to a random process, select some auction, and bid for a product with a probability that may depend on the current value that has been attained by that product. Customers leave the marketplace if they are successful in purchasing the product, and may go to some other auction (or may leave the market) if they are unsuccessful. This analysis leads to a closed form expression for the equilibrium price of all the products in the market, jointly with the number of customers that are present at each of the auctions.

Thus, both the theoretical developments and the potential for new applications have played an important role in the development of G-Networks.

This special issue includes papers which represent both of these trends toward more theory and also broader applications, and we list them in the following order:

(1) "Random Neural Network Methods and Deep Learning," by Yonghua Yin.

(2) "HRNN4F: Hybrid Deep Random Neural Network for Multi-Channel Fall Activity," by Hadi Larijani, Ahsen Tahir, Jawad Ahmed, Gordon Morison, Dawn Skelton and Ryan Gibson.

(3) "Accurate, Energy-Efficient Classification with Spiking Random Neural Networks," by Mohamed Mahdy, Khaled Hussain and Erol Gelenbe.

(4) "G-Networks and the Optimization of Supply Chains," by Yi Wang.

(5) "Optimal Energy Distribution with Energy Packet Networks," by Yunxiao Zhang.

(6) "Energy Packet Networks with Multiple Energy Packets," by Josu Doncel and JeanMichel Fourneau.

(7) "A Random Access G-Network: Stability, Stable Throughput and Queueing Analysis," by Ioannis Dimitriou and Nikolaos Pappas.

(8) "Equilibrium balking strategies in the repairable $M / M / 1$ G-retrial queue with complete removals," by Shan Gao, Deran Zhang, Hua Dong and Xianchao Wang.

(9) "Finding Non-Stationary State Probabilities of Open Markov Networks with Multiple Resources and Various Features," by Mikhail Matalytski and Dimitry Kopats.

(10) "Product Form Markovian Queueing Systems with Multiple Resources," by Valeriy Naumov, Valeriy and Konstantin Samouylov.

The first three papers cover G-Network applications for Machine Learning. The first paper reviews the use of the Random Neural Network (RNN), which is the simplest form of a G-Network for Deep Learning and offers some comparisons of its computational complexity and learning accuracy, showing its significant advantages over other methods that use convolutional networks or other techniques. The second paper describes the use of an RNN-based 
Deep Learning in a specific application where motion is detected and used to predict or infer that a person is falling. On the other hand, the third paper presents experimental results related to the use of the RNN on several standard applications using the RNN's Gradient Descent LearningAlgorithm [27], providing evidence of the high classification accuracy and low computational cost of this approach. An extended version of this paper that contains pointers to the datasets and software which has been used can be found in [43].

The next two papers both use G-Networks with "signals and batch removals" [13] as a tool for optimizing the income obtained from a supply chain, in paper (4), and for choosing the optimal allocation of energy to different servers so as to minimize the average response time of jobs in (5). This is an original application of G-Networks that is also discussed in [37]. Just like the previous paper, the next paper (6) also discusses G-Networks that represent the flow of energy and of jobs or data packets, both represented by discrete entities, where multiple energy packets can be consumed by a data packet.

The following two papers suggest extensions of G-Network models. In the paper (7), the authors introduce a random access model [6] to represent a service mechanism with collisions and repeated trials, which is modified to include negative customers that can destroy certain other customers, and the model is solved under some restrictive assumptions. On the other hand, in (8) the authors also mix a queue with balking and services with retrials together with the effect of negative customers.

Finally the last two papers discuss extensions to G-Network models and their analytical solutions.

We hope that this rich panoply of problems will provide inspiration to many researchers on the theory and applications of stochastic networks, and also suggest ideas for new approaches for modeling the different practical systems that have been described in this paper.

\section{References}

1. Berl, A., Gelenbe, E., Di Girolamo, M., Giuliani, G., De Meer, H., Dang, M.Q., \& Pentikousis, K. (2010). Energy-efficient cloud computing. The Computer Journal 53(7): 1045-1051.

2. Boxma, O.J. \& Gelenbe, E. (1985). Two symmetric queues with alternating service and switching times, in Models of computer system performance (Proceedings 10th IFIP WG\%. 3 International Symposium on Computer Performance Modelling, Measurement and Evaluation, Paris, France, 19-21 December 1984). North-Holland Publishing Company, pp. 409-431.

3. Brun, O., Wang, L., \& Gelenbe, E. (2016). Big data for autonomic intercontinental overlays. IEEE Journal on Selected Areas in Communications 34(3): 575-583.

4. Brun, O., Yin, Y., Gelenbe, E., Kadioglu, Y.M., Augusto-Gonzalez, J., \& Ramos, M. (2018). Deep learning with dense random neural networks for detecting attacks against iot-connected home environments, in Recent Cybersecurity Research in Europe: Proceedings of the 2018 ISCIS Security Workshop, Imperial College London. Lecture Notes CCIS No. 821, Springer Verlag, Vol. 821.

5. Dobson, S., Denazis, S., Fernández, A., Gaïti, D., Gelenbe, E., Massacci, F., Nixon, P., Saffre, F., Schmidt, N., \& Zambonelli, F. (2006). A survey of autonomic communications. ACM Transactions on Autonomous and Adaptive Systems (TAAS) 1(2): 223-259.

6. Fayolle, G., Gelenbe, E., \& Labetoulle, J. (1977). Stability and optimal control of the packet switching broadcast channel. Journal of the ACM (JACM) 24(3): 375-386.

7. Francois, F. \& Gelenbe, E. (2016). Towards a cognitive routing engine for software defined networks, in 2016 IEEE International Conference on Communications (ICC). IEEE, pp. 1-6.

8. Gelenbe, E. (1989). Random neural networks with negative and positive signals and product form solution. Neural Computation 1(4): 502-510.

9. Gelenbe, E. (1990). Réseaux neuronaux aléatoires stables. Comptes rendus de l'Académie des Sciences. Série 2, Mécanique, Physique, Chimie, Sciences de l'Univers, Sciences de la Terre 310(3): 177-180.

10. Gelenbe, E. (1991). Product-form queueing networks with negative and positive customers. Journal of Applied Probability 28(3): 656-663. 
11. Gelenbe, E. (1993). G-networks by triggered customer movement. Journal of Applied Probability 30(3): $742-748$.

12. Gelenbe, E. (1993). Learning in the recurrent random neural network. Neural Computation 5(1): 154-164.

13. Gelenbe, E. (1993). G-networks with signals and batch removal. Probability in the Engineering and Informational Sciences 7(3): 335-342.

14. Gelenbe, E. (1996). Genetic algorithms with analytical solution, in Proceedings of the 1st annual conference on genetic programming. MIT Press, 1996, pp. 437-443.

15. Gelenbe, E. (1997). A class of genetic algorithms with analytical solution. Robotics and Autonomous Systems 22(1): 59-64.

16. Gelenbe, E. (2007). A diffusion model for packet travel time in a random multihop medium. $A C M$ Transactions on Sensor Networks (TOSN) 3(2): 10.

17. Gelenbe, E. (2007). Dealing with software viruses: a biological paradigm. Information Security Technical Report 12(4): 242-250.

18. Gelenbe, E. (2007). Steady-state solution of probabilistic gene regulatory networks. Physical Review E $76(3): 031903$.

19. Gelenbe, E. (2009). Analysis of single and networked auctions. ACM Transactions on Internet Technology (TOIT) 9(2): 8 .

20. Gelenbe, E. (2009). Steps toward self-aware networks. Communications of the ACM 52(7): 66-75.

21. Gelenbe, E. (2012). Energy packet networks: adaptive energy management for the cloud, in Proceedings of the 2nd International Workshop on Cloud Computing Platforms. ACM, p. 1.

22. Gelenbe, E. (2012). Energy packet networks: Ict based energy allocation and storage. In J.J.P.C. Rodrigues, L. Zhou, M. Chen, \& A. Kailas (eds), Green communications and networking, Berlin, Heidelberg: Springer, pp. 186-195.

23. Gelenbe, E. (2015). Synchronising energy harvesting and data packets in a wireless sensor. Energies 8(1): 356-369.

24. Gelenbe, E. \& Abdelrahman, O.H. (2018). An energy packet network model for mobile networks with energy harvesting. IEICE Nonlinear Theory and Its Applications 9(3): 1-15, doi: 10.1587/nolta.9.1.

25. Gelenbe, E. \& Ceran, E.T. (2015). Central or distributed energy storage for processors with energy harvesting, in The Fourth International Conference on Sustainable Internet and ICT for Sustainability. IEEE, April 2015.

26. Gelenbe, E. \& Ceran, E.T. (2016). Energy packet networks with energy harvesting. IEEE Access 4: 1321-1331.

27. Gelenbe, E. \& Hussain, K. (2002). Learning in the multiple class random neural network. IEEE Transactions on Neural Networks 13(6): 1257-1267. [Online]. Available: http://dx.doi.org/10.1109/TNN.2002. 804228.

28. Gelenbe, E. \& Loukas, G. (2007). A self-aware approach to denial of service defence. Computer Networks 51(5): 1299-1314.

29. Gelenbe, E. \& Mahmoodi, T. (2011). Energy-aware routing in the cognitive packet network, in ENERGY 2011, The First International Conference on Smart Grids, Green Communications and IT Energyaware Technologies, pp. 7-12.

30. Gelenbe, E. \& Morfopoulou, C. (2011). A framework for energy-aware routing in packet networks. The Computer Journal 54(6): 850-859.

31. Gelenbe, E. \& Muntz, R.R. (1976). Probabilistic models of computer systems: Part i (exact results). Acta Informatica 7(1): 35-60.

32. Gelenbe, E. \& Pujolle, G. (1982). Introduction aux réseaux de files d'attente, Paris: Eyrolles.

33. Gelenbe, E. \& Sevcik, K. (1979). Analysis of update synchronization for multiple copy data bases. IEEE Transactions on Computers 28(10): 737-747.

34. Gelenbe, E. \& Stafylopatis, A. (1991). Global behavior of homogeneous random neural systems. Applied Mathematical Modelling 15(10): 534-541.

35. Gelenbe, E. \& Wu, F.-J. (2013). Future research on cyber-physical emergency management systems. Future Internet 5(3): 336-354.

36. Gelenbe, E. \& Yin, Y. (2016). Deep learning with random neural networks, in 2016 International Joint Conference on Neural Networks (IJCNN). IEEE, pp. 1633-1638.

37. Gelenbe, E. \& Zhang, Y. (2019). IEEE Systems Journal, pp. 1-11.

38. Gelenbe, E., Glynn, P., \& Sigman, K. (1991). Queues with negative arrivals. Journal of Applied Probability 28(1): 245-250.

39. Gelenbe, E., Mang, X., \& Önvural, R. (1996). Diffusion based statistical call admission control in atm. Performance Evaluation 27: 411-436. 
40. Gelenbe, E., Lent, R., \& Nunez, A. (2004). Self-aware networks and qos. Proceedings of the IEEE 92(9): 1478-1489.

41. Gelenbe, E., Liu, P., \& Lainé, J. (2006). Genetic algorithms for route discovery. IEEE Transactions on Systems, Man, and Cybernetics, Part B: Cybernetics 36(6): 1247-1254.

42. Gorbil, G. \& Gelenbe, E. (2011). Opportunistic communications for emergency support systems. Procedia Computer Science 5: 39-47.

43. Hussain, K.F., Bassyouni, M.Y., \& Gelenbe, E. (2019). Accurate and energy-efficient classification with spiking random neural network: Corrected and expanded version, Arxiv.

44. Kadioglu, Y.M. \& Gelenbe, E. (2018). Product-form solution for cascade networks with intermittent energy. IEEE Systems Journal 99: 1-10.

45. Kim, H. \& Gelenbe, E. (2010). Stochastic gene expression model base gene regulatory networks, in $E K C$ 2009 Proceedings of the EU-Korea Conference on Science and Technology. Springer Berlin Heidelberg, pp. 235-244.

46. Kim, H. \& Gelenbe, E. (2012). Stochastic gene expression modeling with hill function for switch-like gene responses. IEEE/ACM Transactions on Computational Biology and Bioinformatics 9(4): 973-979, [Online]. Available: http://doi.ieeecomputersociety.org/10.1109/TCBB.2011.153.

47. Kim, H. \& Gelenbe, E. (2012). Reconstruction of large-scale gene regulatory networks using bayesian model averaging. IEEE Transactions on NanoBioscience 11(3): 259-265.

48. Kim, H., Atalay, R., \& Gelenbe, E. (2011). G-network modelling based abnormal pathway detection in gene regulatory networks, in Computer and Information Sciences: 26th International Symposium on Computer and Information Sciences. Springer Verlag, p. 257.

49. Kim, H., Park, T., \& Gelenbe, E. (2014). Identifying disease candidate genes via large-scale gene network analysis. International Journal of Data Mining and Bioinformatics 10(2): 175-188.

50. Oke, G., Loukas, G., \& Gelenbe, E. (2007). Detecting denial of service attacks with bayesian classifiers and the random neural network, in 2007 IEEE International Fuzzy Systems Conference. IEEE, pp. 1-6.

51. Pernici, B., Aiello, M., vom Brocke, J., Donnellan, B., Gelenbe, E., \& Kretsis, M. (2012). What is can do for environmental sustainability: a report from caise2011 panel on green and sustainable is. Communications of the Association for Information Systems 30(1): 18.

52. Phan, H., Sterberg, M.J., \& Gelenbe, E. (2012). Aligning protein-protein interaction networks using random neural networks. Bioinformatics and Biomedicine (BIBM), 2012 IEEE International Conference on IEEE, pp. 1-6.

53. Walrand, J. (1998). An introduction to queueing networks, Englewood Cliffs, N.J.: Prentice Hall.

54. Wang, L. \& Gelenbe, E. (2018). Adaptive dispatching of tasks in the cloud. IEEE Transactions on Cloud Computing 6(1): 33-45.

55. Wang, L., Brun, O., \& Gelenbe, E. (2016). Adaptive workload distribution for local and remote clouds, in 2016 IEEE International Conference on Systems, Man, and Cybernetics (SMC). IEEE, pp. $003984-$ 003988 . 